\title{
Existence and Uniqueness of Solution to Two-Point Boundary Value for Two-Sided Fractional Differential Equations
}

\author{
Ailing Shi, Yu Bai \\ School of Science, Beijing University of Civil Engineering and Architecture, Beijing, China \\ Email: shiailing@bucea.edu.cn
}

Received November 28, 2012; revised March 1, 2013; accepted March 9, 2013

Copyright (C) 2013 Ailing Shi, Yu Bai. This is an open access article distributed under the Creative Commons Attribution License, which permits unrestricted use, distribution, and reproduction in any medium, provided the original work is properly cited.

\begin{abstract}
In this paper, existence and uniqueness of solution to two-point boundary value for two-sided fractional differential equations involving Caputo fractional derivative is discussed, by means of the Min-Max Theorem.
\end{abstract}

Keywords: Existence; Uniqueness; Two-Sided Fractional Differential Equations

\section{Introduction}

In this paper, using the Min-Max Theorem, we will devote to considering the existence and uniqueness result of solution to the following two-sided fractional differential equations boundary value problems (BVP for short)

$$
\left\{\begin{array}{l}
D_{T-}^{\alpha}\left(D_{0+}^{\alpha} u(t)\right)=g(t, u), \quad t \in[0, T], 0<T<\infty, \\
u(0)=a, u(T)=b,
\end{array}\right.
$$

where $D_{T-}^{\alpha}, D_{0+}^{\alpha}$ denote the right-side and left-side Caputo fractional derivative of order $0<\alpha<1$, respectively, $g:[0, T] \times R \rightarrow R$ is a continuous differential function with respect to all variables, and $a, b \in R$.

In particular, if $\alpha=1$, BVP (1.1) reduces to the standard second order boundary value problem of the following form

$$
\left\{\begin{array}{l}
u^{\prime \prime}+g(t, u)=0, \quad t \in[0, T], 0<T<\infty, \\
u(0)=a, u(T)=b .
\end{array}\right.
$$

Recently, fractional differential equations have been verified to be valuable tools in the modeling of many phenomena in various fields of science and engineering. There have many papers which are concerning with the existence of solutions for fractional differential equations boundary value problems, by means of some classic fixed point theorems and monotone iterative methods, such as [1-11], etc. But, as far as we known, there are few papers which considered the existence of solutions for fractional differential equations boundary value problems using the variable method, such as the direct method, the critical point theory. Recently, there appeared some interesting works $[12,13]$ considering existence of solution to fractional differential problems, by means of the variable way, In [13], by the critical point theory, author considered the existence of solutions of the following a twopoint boundary value problem for some class of fractional differential equation containing the left and right Riemann-Liouville fractional derivative operators

$$
\left\{\begin{array}{l}
{ }_{t} D_{T}^{\alpha}\left({ }_{0} D_{t}^{\alpha} u(t)\right)=\nabla F(t, u(t)), \text { a.e. } t \in(0, T], \\
u(0)=u(T)=0,
\end{array}\right.
$$

where ${ }_{t} D_{T}^{\alpha}$ and ${ }_{0} D_{t}^{\alpha}$ are the right and left RiemannLiouville fractional derivatives of order $0<\alpha \leq 1$ respectively, $F:[0, T] \times R^{N} \rightarrow R$ is a given function satisfying some assumptions and $\nabla F(t, x)$ is the gradient of $F$ at $x$. This is a very interesting and meaning works, this is the first time that the existence of solutions for fractional differential equation two-point boundary value problem via the critical point theory.

The following are definitions and some properties of Riemann-Liouville fractional integral and derivative, the Caputo fractional derivative, for the details, please see [1].

The left Riemann-Liouville fractional integrals (LFLI) of order $\beta>0$ of function $f(t)$ which is defined as follows,

$$
I_{0+}^{\beta} f(t)=\frac{1}{\Gamma(\beta)} \int_{0}^{t}(t-s)^{\beta-1} f(s) \mathrm{d} s, t>0
$$


The right Riemann-Liouville fractional integrals (RFLI) of order $\beta>0$ of function $f(t)$ which is defined as follows,

$$
I_{T-}^{\beta} f(t)=\frac{1}{\Gamma(\beta)} \int_{t}^{T}(s-t)^{\beta-1} f(s) \mathrm{d} s, t>0
$$

The left Riemann-Liouville fractional derivative (LFLD) of order $0<\gamma<1$ of function $f(t)$ which is defined as follows,

$$
{ }_{0} D_{t}^{\gamma} f(t)=\frac{\mathrm{d}}{\mathrm{d} t} I_{0+}^{1-\gamma} f(t), t>0
$$

The right Riemann-Liouville fractional derivative (RFLD) of order $0<\gamma<1$ of function $f(t)$ which is defined as follows,

$$
{ }_{t} D_{T}^{\gamma} f(t)=-\frac{\mathrm{d}}{\mathrm{d} t} I_{T-}^{1-\gamma} f(t), t>0
$$

The left Caputo fractional derivative (LCFD) of order $0<\gamma<1$ of function $f(t)$ which is defined as follows,

$$
D_{0+}^{\gamma} f(t)={ }_{0} D_{t}^{\gamma} f(t)-\frac{f(0)}{\Gamma(1-\gamma)} t^{-\gamma}, t>0
$$

The right Caputo fractional derivative (RCFD) of order $0<\gamma<1$ of function $f(t)$ which is defined as follows,

$$
D_{T-}^{\gamma} f(t)={ }_{t} D_{T}^{\gamma} f(t)-\frac{f(T)}{\Gamma(1-\gamma)}(T-t)^{-\gamma}, t>0
$$

Remark 1.1. Obviously, if $f(a)=0$, then ${ }_{0} D_{t}^{\gamma} f(t)=D_{0+}^{\gamma} f(t)$; it $f(T)=0$, then ${ }_{t} D_{T}^{\gamma} f(t)=D_{T-}^{\gamma} f(t)$.

It is well known that there are several kinds of fractional derivatives, such as, Riemann-liouville fractional derivative, Marchaud fractional derivative, Caputo derivative, Griinwald-Letnikov fractional derivative, etc. Since as cited in [2] there have appeared a number of works, especially in the theory of viscous elasticity and in hereditary solid mechanics, where fractional derivatives are used for a better description of material properties. Mathematical modeling based on enhanced rheological models naturally leads to differential equations of fractional order and to the necessity of the formulation of initial conditions to such equations. Applied problems require definitions of fractional derivatives allowing the utilization of physically in interpretable initial conditions, which contain $f(a), f^{\prime}(a)$, etc". In fact, the same requirements apply for boundary conditions. Therefore, we cannot impose initial and boundary conditions, such as $u(0)=d \neq 0, u(T)=e$ on problems involving the Riemann-Liouville fractional derivative ${ }_{0} D_{t}^{\alpha}$ or ${ }_{t} D_{t}^{\alpha}$. We find that Caputo fractional derivative exactly satisfies these demands. Therefore in this article, we deal with boundary value problem for fractional differential equation involving Caputo derivative.

The following is the rule of fractional integration by parts for LFLI and RFLI.

Let $0<\alpha<1, \quad p \geq 1, q \geq 1$, and $\frac{1}{p}+\frac{1}{q} \leq 1+\alpha$. If $g \in L_{p}[0, T], f \in L_{q}[0, T]$, then

$$
\int_{0}^{T} g(t) I_{0+}^{\alpha} f(t) \mathrm{d} t=\int_{0}^{T} f(t) I_{T-}^{\alpha} g(t) \mathrm{d} t
$$

We let $0<\gamma<1, \quad p \geq 1, q \geq 1$, and $\frac{1}{p}+\frac{1}{q} \leq 1+\gamma$, if $f \in L_{p}[0, T], f(0)=0, D_{0+}^{\gamma} f \in L_{p}[0, T]$, $g \in L_{q}[0, T], g(T)=0, D_{T-}^{\gamma} g \in L_{q}[0, T]$, then, by (1.9) and Remark 1.1, we have that

$$
\int_{0}^{T} g(t) D_{0+}^{\gamma} f(t) \mathrm{d} t=\int_{0}^{T} f(t) D_{T-}^{\gamma} g(t) \mathrm{d} t
$$

Inspired by $[12,13]$, in this paper, we will consider the unique existence of solution to problem (1.1), by means of the following Min-Max Theorem.

Min-Max Theorem (Manasevich). [14] Let $H$ be a real Hilbert space and let $f: H \rightarrow R$ be of class $C^{2}$. Suppose that there exist two closed subspaces $X$ and $Y$ such that $H=X \oplus Y$ and two continuous non-increasing functions $\zeta:[0, \infty) \rightarrow(0, \infty), \quad \eta:[0, \infty) \rightarrow(0, \infty)$ such that

$$
\begin{gathered}
\int_{1}^{\infty} \zeta(s) \mathrm{d} s=\infty, \quad \int_{1}^{\infty} \eta(s) \mathrm{d} s=\infty, \\
\left\langle D^{2} f(x+y) k, k\right\rangle \geq \zeta(y) k^{2}
\end{gathered}
$$

for all $x \in X, y \in Y$ and $k \in Y$, and

$$
\left\langle D^{2} f(x+y) h, h\right\rangle \leq-\eta(x) h^{2}
$$

for all $x \in X, y \in Y$ and $h \in X$. Then

1) there exists a unique $v_{0} \in H$ such that $\nabla f\left(v_{0}\right)=0$;

$$
\text { 2) } \begin{aligned}
f\left(v_{0}\right) & =\max _{x \in X} \min _{y \in Y} f(x+y) \\
& =\min _{y \in Y} \max _{x \in X} f(x+y) .
\end{aligned}
$$

Here, $\nabla f$ and $D^{2} f$ denote the gradient and the Hessian of $f$ at $u \in H$, respectively. In this case, $f: H \rightarrow R$ is a $C^{\prime}$ mapping and $(\nabla f)^{\prime} u=D^{2} f$ is a bounded self-adjoins linear operator on $H$.

\section{Basic Facts}

In [13], authors gave the definition of solution to (1.2) as following

Definition 2.1. [13] A function $u:[0, T] \rightarrow R^{N}$ is called a solution of BVP (1.2) if

(i) ${ }_{t} D_{T}^{\alpha-1}\left({ }_{0} D_{t}^{\alpha} u(t)\right)$ and ${ }_{0} D_{t}^{\alpha-1} u(t)$ are derivative for almost every $t \in[0, T]$, and (ii) $u$ satisfies (1.2).

In [13], in order to establish a variational structure 
which enables ones to reduce the existence of solutions of BVP (1.2) to the one of critical points of corresponding functional, authors constructed an appropriate function spaces $E_{0}^{\alpha, p}$, which depend on $L_{p}$-integrability of the Riemann-Liouville fractional derivative of a function.

Definition 2.2. [13] Let $0<\alpha \leq 1,1<p<+\infty$. The fractional derivative space $E_{0}^{\alpha, p}$ is defined by the closure of $C_{0}^{\infty}\left([0, T], R^{N}\right)$ with respect to the norm

$$
\|u\|_{E_{0}^{\alpha, p}}=\left(\int_{0}^{T}|u(t)|^{p} \mathrm{~d} t+\int_{0}^{T}\left|{ }_{0} D_{t}^{\alpha} u(t)\right|^{p} \mathrm{~d} t\right)^{\frac{1}{p}} .
$$

It is obvious that space $E_{0}^{\alpha, p}$ is the space of functions $u \in L_{p}\left([0 ; T] ; R^{N}\right)$ having an $\alpha$-order fractional derivative ${ }_{0} D_{t}^{\alpha} u(t) \in L_{p}\left([0, T] ; R^{N}\right)$ and $u(0)=u(T)=0$. Furthermore, it is easy to verify that $E_{0}^{\alpha, p}$ is a reflexive and separable Banach space.

Theorem 2.3. [13] Let $0<\alpha \leq 1,1<p<+\infty$. The space $E_{0}^{\alpha, p}$ is a reflexive and separable Banach space.

Proposition 2.4. [13] Let $0<\alpha \leq 1,1<p<+\infty$. For all $u \in E_{0}^{\alpha, p}$, if $1-\alpha \geq \frac{1}{p}$ or $\alpha>\frac{1}{p}$, we have

$$
u_{L_{p}} \leq \frac{T^{\alpha}}{\Gamma(1+\alpha)_{0}} D_{t}^{\alpha} u(t)_{L_{p}} .
$$

with this property, one can consider $E_{0}^{\alpha, p}$ with respect to the norm

$$
u_{E_{0}^{\alpha, p}}=\left(\int_{0}^{T}\left|{ }_{0} D_{t}^{\alpha} u(t)\right|^{p} \mathrm{~d} t\right)^{\frac{1}{p}} .
$$

If $\alpha>1 / p$, the following theorem is useful for us to establish the variational structure on the space $E_{0}^{\alpha, p}$ for BVP (1.2).

Theorem 2.5. [13] Let $1<p<+\infty, 1 / p<\alpha<1$ and $L:[0 ; T] \times R^{N} \times R^{N} \rightarrow R, \quad(t ; x ; y) \rightarrow L(t ; x ; y)$ be measurable in $t$ for each $[x ; y] \in R^{N} \times R^{N}$ and continuously differentiable in $[x ; y]$ for almost every $t \in[0, T]$. If there exists $m_{1} \in C\left(R^{+} ; R^{+}\right) ; \quad m_{2} \in L_{1}\left([0 ; T] ; R^{+}\right)$and $m_{3} \in L_{q}\left([0 ; T] ; R^{+}\right) ; 1<q<1$, such that, for a.e. $t \in[0 ; T]$ and every $[x ; y] \in R^{N} \times R^{N}$, one has

$$
\begin{gathered}
|L(t ; x ; y)| \leq m_{1}(|x|)\left(m_{2}(t)+|y|^{p}\right) ; \\
\left|D_{x} L(t ; x ; y)\right| \leq m_{1}(|x|)\left(m_{2}(t)+|y|^{p}\right) ; \\
\left|D_{y} L(t ; x ; y)\right| \leq m_{1}(|x|)\left(m_{3}(t)+|y|^{p-1}\right) ;
\end{gathered}
$$

where $\frac{1}{p}+\frac{1}{q}=1$, then the functional defined by

$$
\phi(u)=\int_{0}^{T} L\left(t, u(t),{ }_{0} D_{t}^{\alpha} u(t)\right) \mathrm{d} t
$$

is continuously differentiable on $E_{0}^{\alpha, p}$, and $\forall u, v \in E_{0}^{\alpha, p}$, we have

$$
\begin{aligned}
\left\langle\phi^{\prime}(u), v\right\rangle= & \int_{0}^{T}\left[\left(D_{x} L\left(t, u(t),{ }_{0} D_{t}^{\alpha} u(t)\right), v(t)\right)\right. \\
& \left.+\left(D_{y} L\left(t, u(t),{ }_{0} D_{t}^{\alpha} u(t)\right),{ }_{0} D_{t}^{\alpha} v(t)\right)\right] \mathrm{d} t .
\end{aligned}
$$

From, we known that, for a solution $u \in E^{\alpha}=: E_{0}^{\alpha, 2}$ of BVP (1.2) such that $\nabla F(\cdot ; u(\cdot)) \in L_{1}\left([0 ; T] ; R^{N}\right)$, multiplying (1.2) by $v \in C_{0}^{1}\left([0 ; T] ; R^{N}\right)$ yields

$$
\begin{aligned}
& \int_{0}^{T}\left[\left({ }_{t} D_{T}^{\alpha}\left({ }_{0} D_{t}^{\alpha} u(t)\right), v(t)\right)-(\nabla F(t, u(t)), v(t))\right] \mathrm{d} t \\
& =\int_{0}^{T}\left[\left({ }_{0} D_{t}^{\alpha} u(t),{ }_{0} D_{t}^{\alpha} v(t)\right)-(\nabla F(t, u(t)), v(t))\right] \mathrm{d} t \\
& =0 .
\end{aligned}
$$

According these facts, authors [13] gave the definition of weak solution for BVP (1.2) as follows.

Definition 2.6. [13] By the weak solution of BVP (1.2), we mean that the function $u \in E^{\alpha}$ such that $\nabla F(\cdot ; u(\cdot)) \in L_{1}\left([0 ; T] ; R^{N}\right)$ and satisfies the above equality for all $v \in C_{0}^{1}\left([0 ; T] ; R^{N}\right)$.

Using the direct method and the Mountain pass theorem, authors obtain two existence results of weak solution to (1.2), please see [13].

Basing on some deductions, authors verified that a weak of (1.2) is also its solution.

\section{Main Result}

From the Remark 1.1 and Definition 2.2, we will use function space $E^{\alpha}$ in the following arguments.

Theorem 3.1. Assume that $g:[0, T] \times R \rightarrow R$ is continuous differentiable with respect to its two variables, there is a constant $0<a<\frac{\Gamma(1+\alpha)}{T^{\alpha}}$ such that $g_{u}(t, u) \leq a$ for all $t \in[0, T], u \in R$. Then problem (1.1) exists unique solution $u \in E^{\alpha}$.

Proof. We can decompose (1.1) into the following two problems

$$
\begin{aligned}
& \left\{\begin{array}{l}
D_{T-}^{\alpha}\left(D_{0+}^{\alpha} u(t)\right)=g(t, u), \quad t \in[0, T], 0<T<\infty, \\
u(0)=0, u(T)=0,
\end{array}\right. \\
& \left\{\begin{array}{l}
D_{T-}^{\alpha}\left(D_{0+}^{\alpha} u(t)\right)=0, \quad t \in[0, T], 0<T<\infty, \\
u(0)=a, u(T)=b,
\end{array}\right.
\end{aligned}
$$

according to the linearity of $D_{T-}^{\alpha}$ and $D_{0+}^{\alpha}$, we can easily know that if $u_{1}, u_{2}$ are solution of (3.1), (3.2), respectively, then $u=u_{1}+u_{2}$ is a solution of (1.1). Obviously, $u_{2}(t)=a+\frac{(b-a) \Gamma(1+\alpha)}{T^{\alpha} \Gamma(1+\alpha)} t^{\alpha} \quad$ is unique solution of (3.2). Next, we will verify that (3.1) exists unique 
solution $u_{1} \in E^{\alpha}$, by means of the Min-Max Theorem (Manasevich).

From [13], we know that $E^{\alpha}$ is a real Hilbert space with the inner product by

$$
\langle u, v\rangle=\int_{0}^{T} D_{0+}^{\alpha} u(t) D_{0+}^{\alpha} v(t) \mathrm{d} t .
$$

It follows from assumptions on function $g$ that we can easily know that $g$ satisfies assumption of Theorem 2.5 .

We let $X=\{0\}, Y=E^{\alpha}$, clearly, we have

$E^{\alpha}=X \oplus Y$. From the Algebra knowledge, it is well know that $Y=E^{\alpha}$ and $X=\{0\}$ are closed subsets of $E^{\alpha}$. From the previous arguments, we can complete this proof through two steps.

The first step, we will consider the existence of critical point of functional defined as following

$$
\phi(u)=\frac{1}{2} \int_{0}^{T}\left|D_{0+}^{\alpha} u(t)\right|^{2} \mathrm{~d} t-\int_{0}^{T} G(t, u) \mathrm{d} t, u \in E^{\alpha},
$$

where $G(t, u)=\int_{0}^{u} g(t, s) \mathrm{d} s, t \in[0, T]$. From the arguments in [13], we know that

$$
\begin{aligned}
\langle\phi(u), v\rangle & =\int_{0}^{T} D_{0+}^{\alpha} u(t) D_{0+}^{\alpha} v(t) \mathrm{d} t \\
& -\int_{0}^{T} g(t, u) v(t) \mathrm{d} t,
\end{aligned}
$$

for $u, v \in E^{\alpha}$. By the assumptions and the analogy arguments with, we have that

$$
\begin{aligned}
\left\langle D^{2} \phi(u) w, v\right\rangle & =\int_{0}^{T} D_{0+}^{\alpha} w(t) D_{0+}^{\alpha} v(t) \mathrm{d} t \\
& -\int_{0}^{T} g_{u}(t, u) w(t) v(t) \mathrm{d} t,
\end{aligned}
$$

for $u, v, w \in E^{\alpha}$.

For all $x \in X, y \in Y$ and $k \in Y$, by Proposition 2.4, we have that

$$
\begin{aligned}
& \left\langle D^{2} \phi(x+y) k, k\right\rangle \\
& =\int_{0}^{T}\left|D_{0+}^{\alpha} k(t)\right|^{2} \mathrm{~d} t-\int_{0}^{T} g_{u}(t, u) k^{2} \mathrm{~d} t \\
& \geq \int_{0}^{T}\left|D_{0+}^{\alpha} k(t)\right|^{2} \mathrm{~d} t-a \int_{0}^{T} k^{2} \mathrm{~d} t \\
& \geq \int_{0}^{T}\left|D_{0+}^{\alpha} k(t)\right|^{2} \mathrm{~d} t-\frac{a T^{\alpha}}{\Gamma(1+\alpha)} \int_{0}^{T}\left|D_{0+}^{\alpha} k(t)\right|^{2} \mathrm{~d} t \\
& =\left(1-\frac{a T^{\alpha}}{\Gamma(1+\alpha)}\right) \int_{0}^{T}\left|D_{0+}^{\alpha} k(t)\right|^{2} \mathrm{~d} t=\zeta(\|y\|)\|k\|_{E^{\alpha}} .
\end{aligned}
$$

For all $x \in X, y \in Y$ and $h \in X$, we have that

$$
0=\left\langle D^{2} \phi(x+y) h, h\right\rangle=0
$$

which implies that

$$
\left\langle D^{2} \phi(x+y) h, h\right\rangle \leq-\eta(\|x\|)\|h\|_{E^{\alpha}}
$$

holds for all $x \in X, y \in Y$ and $h \in X$. Obviously, func- tions $\zeta(t)=1-\frac{a T^{\alpha}}{\Gamma(1+\alpha)}, \eta(t)=1$ satisfy assumption conditions of the Min-Max Theorem. Hence, the MinMax Theorem assures that there exists unique $u_{1} \in E_{\alpha}$ such that $\nabla \phi\left(u_{1}\right)=0$, which means that $u_{1}$ is a unique weak solution of (3.1).

It follows from $u_{1} \in E^{\alpha}$ that $u_{1}(0)=0$, hence the left Caputo fractional equal to the left Riemann-Liouville fractional derivative. Hence, by the similar proofs of lemma theorem, we know that this weak solution $u_{1} \in E_{\alpha}$ is also a solution of (3.1). Thus, we obtain that $u=u_{1}+u_{2}$ is unique solution of (1.1).

\section{Conclusion}

In this paper, using a Min-Max Theorem (Manasevich), we considered the existence and uniqueness of solution to some class of two-sided fractional differential equations with two-point boundary value problems.

\section{Acknowledgements}

The authors would like to thank those listed references for their helpful suggestions, which helped to improve the quality of the paper. This research supported by 2013 Science and Technology Research Project of Beijing Municipal Education Commission (KM201310016001) and 2011 Science and Technology Research Project of Beijing Municipal Education Commission (KM2011100160 12).

\section{REFERENCES}

[1] A. A. Kilbas, H. M. Srivastava and J. J. Trujillo, "Theory and Applications of Fractional Differential Equations," Elsevier, Amsterdam, 2006.

[2] I. Podlubny, "Fractional Differential Equations," In: Mathematics in Science and Engineering, Academic Press, San Diego, 1999.

[3] D. Delbosco and L. Rodino, "Existence and Uniqueness for a Nonlinear Fractional Differential Equation,” Journal of Mathematical Analysis and Applications, Vol. 204, No. 2, 1996, pp. 609-625. doi:10.1006/jmaa.1996.0456

[4] V. Lakshmikantham and A. S. Vatsala, "Theory of Fractional Differential Inequalities and Applications,” Communications on Pure and Applied Analysis, Vol. 11, No. 3-4, 2007, pp. 395-402.

[5] V. Lakshmikantham and A. S. Vatsala, "General Uniqueness and Monotone Iterative Technique for Fractional Differential Equations," Applied Mathematics Letters, Vol. 21, No. 8, 2008, pp. 828-834. doi:10.1016/j.aml.2007.09.006

[6] V. Lakshmikantham and A. S. Vatsala, "Basic Theory of Fractional Differential Equations," Nonlinear Analysis: Theory, Methods \& Applications, Vol. 69, No. 8, 2008, pp. 2677-2682. doi:10.1016/j.na.2007.08.042 
[7] H. Liang and J. H. Zhang, "Positive Solutions for Boundary Value Problems of Nonlinear Fractional Differential Equation,” Nonlinear Analysis: Theory, Methods \& Applications, Vol. 71, No. 11, 2009, pp. 5545-5550. doi:10.1016/j.na.2009.04.045

[8] S. Q. Zhang, "Positive Solutions to Singular Boundary Value Problem for Nonlinear Fractional Differential Equation," Computers \& Mathematics with Applications, Vol. 59, No. 3, 2010, pp. 1300-1309. doi:10.1016/j.camwa.2009.06.034

[9] Z. Bai and H. Lu, "Positive Solutions for Boundary Value Problem of Nonlinear Fractional Differential Equation," Journal of Mathematical Analysis and Applications, Vol. 311, No. 2, 2005, pp. 495-505. doi:10.1016/j.jmaa.2005.02.052

[10] R. P. Agarwal, Y. Zhou, J. R. Wang and X. N. Luo, "Fractional Functional Differential Equations with Causal Operators in Banach Spaces," Mathematical and Com- puter Modelling, Vol. 54, No. 5-6, 2011, pp. 1440-1452. doi:10.1016/j.mcm.2011.04.016

[11] F. L. Chen, J. J. Nieto and Y. Zhou, "Global Attractivity for Nonlinear Fractional Differential Equations," Nonlinear Analysis: Real World Applications, Vol. 13, No. 1, 2012, pp. 287-298. doi:10.1016/j.nonrwa.2011.07.034

[12] F. Jiao and Y. Zhou, "Existence of Solutions for a Class of Fractional Boundary Value Problems via Critical Point theory," Computers \& Mathematics with Applications, Vol. 62, No. 3, 2011, pp. 1181-1199. doi:10.1016/j.camwa.2011.03.086

[13] F. Jiao and Y. Zhou, "Existence of Solutions for a Class of Fractional Boundary Value Problems via Critical Point Theory,” International Journal of Bifurcation and Chaos, Special Issue, to Appear.

[14] R. F. Manasevich, “A Min-Max Theorem,” Journal of Mathematical Analysis and Applications, Vol. 90, No. 1, 1982, pp. 64-71. doi:10.1016/0022-247X(82)90044-0 